\title{
Diagnóstico situacional de los laboratorios clínicos del Ecuador: Análisis de la gestión en estos servicios
}

\author{
Anabel Burbano García ${ }^{1,}$ Benjamín Puertas Donoso ${ }^{2}$ \\ 'Profesora, Universidad Central del Ecuador, Quito-Ecuador. \\ ${ }^{2}$ Director de la Maestría en Salud Pública, Universidad San Francisco de Quito, Ecuador
}

\begin{abstract}
RESUMEN.- El presente es un diagnóstico situacional de los laboratorios clínicos del país, que busca evaluar el sistema de registro de los mismos, el recurso humano que allí labora y su idoneidad. Para el diagnóstico se consideró a todas las 21 provincias del país a las que se envió un Formulario de Recolección de Datos de Laboratorio y se pidió la información de los Catastros respectivos en el lapso de un año. Se realizó un seguimiento a través de llamadas telefónicas a las Direcciones Provinciales de Salud.

Apenas nueve de las 21 provincias entregaron la información catastral y 14 provincias los Formularios de Recolección de Datos. Solo cinco provincias enviaron la información tanto de Catastro como del Formulario. Carchi, Napo y Morona Santiago no enviaron ningún tipo de información. Ninguna provincia proporcionó información catastral completa. Pichincha reportó el mayor número de laboratorios (78\%). De un total de 1001 laboratorios registrados en los Formularios, 452 (46\%) no contaban con permiso de funcionamiento. En Los Ríos, Pastaza, Esmeraldas, y Guayas, el porcentaje de laboratorios sin permiso de funcionamiento fue igual o mayor a $50 \%$. Se observó una diferencia de
\end{abstract}

110 laboratorios (33\%) entre los registrados en Catastro (329) y los laboratorios con permiso de funcionamiento que constan en los Formularios (219), siendo esta diferencia más notoria en algunas provincias. Los laboratorios clínicos ubicados en servicios de salud privados (93) representaron el $76,2 \%$ del total de los laboratorios con permiso de funcionamiento. Guayas tuvo el mayor porcentaje de profesionales con el perfil adecuado para manejar laboratorios clínicos $(24,6 \%)$, seguido por Pichincha $(24,3 \%)$.

El estudio evidenció la falta de regulación de los laboratorios clínicos en las provincias investigadas, al igual que una serie de omisiones importantes (infraestructura y recurso humano idóneo). Muchos de los laboratorios clínicos en las diferentes provincias participantes no contaban con permiso de funcionamiento. Existen laboratorios en servicios de salud privados que posiblemente no cuentan con el respectivo permiso. En general, los bioquímicos fueron los profesionales con perfil adecuado a cargo de la mayoría de laboratorios clínicos de las provincias del estudio, aunque preocupa el hecho de que muchos laboratorios eran manejados por personal de apoyo. No existe un sistema de información adecuado 
y se evidencia una falta de registro de laboratorios clínicos. El MSP no cuenta con una red de información intrainstitucional eficiente y no existe un adecuado control y vigilancia de los laboratorios clínicos ubicados dentro de servicios privados. Se evidencia un incumplimiento del marco legal en relación al buen funcionamiento de los laboratorios.

Es recomendable implementar un sistema de información automatizado en las Direcciones Provinciales de Salud y una red de información intrainstitucional. Se recomienda un censo de laboratorios clínicos en el Ecuador. Debe darse un proceso de revisión de los desfases en los niveles correspondientes y se debe garantizar la entrega de información por parte de todas las provincias. Es importante analizar, evaluar y mejorar el actual reglamento de funcionamiento de los laboratorios clínicos y que todos tengan el respectivo permiso de funcionamiento. Debido a la carencia de personal adecuado a cargo de laboratorios clínicos, se debería estudiar la posibilidad de un año de trabajo rural para los bioquímicos y químicos farmacéuticos que egresan de la facultad del país.

\section{INTRODUCCIÓN}

El actual modelo de atención integral de servicios de salud se enmarca en las reformas que dispone la Ley del Sistema Nacional de Salud. ${ }^{1}$ Dentro de la propuesta de Rectoría del Estado en Salud se incluye la garantía de calidad y control sanitario de los prestadores de salud, entre los que constan los laboratorios clínicos públicos y privados. ${ }^{2}$ El Código de la Salud, marco legal sanitario, expidió el Reglamento para el Funcionamiento de los Laboratorios de Diagnóstico Clínico el 27 de mayo de 1998, aplicable al sistema de atención privada y pública. En él se incluyen los requisitos para controlar y evaluar periódicamente a los laboratorios clínicos, así como para vigilar el ejercicio de las profesiones médicas, afines y convexas. ${ }^{3}$ Es importante señalar que los requisitos establecidos en esta normativa cumplen los parámetros necesarios, pero los niveles de vigilancia del cumplimiento no han sido lo suficientemente estrictos. ${ }^{4}$

La vigilancia de la salud individual y colectiva, el desarrollo de un número creciente de técnicas de laboratorio y la mejora permanente de los métodos diagnósticos, requieren "la adopción de eficaces herramientas de gestión"s para su operacionalización a nivel de sistemas y laboratorios de salud. De acuerdo a Gabastou, asesor en Servicios de Laboratorios de Salud Pública de la OPS para la región, "Los laboratorios juegan un papel fundamental. De la calidad de los servicios de laboratorio dependerá la calidad de la información para la toma oportuna de decisiones". 6

En la actualidad existe un sistema de información en el que se incluye datos de los laboratorios clínicos del sector público. Esto tiene un rol determinante en el Sistema de Vigilancia Epidemiológica del Ecuador (SIVE), pues constituye un elemento importante en el proceso de prevención, diagnóstico y tratamiento de las enfermedades. Cabe mencionar que en dicho sistema aún no se integra la información proveniente de los laboratorios clínicos privados del Ecuador, y tampoco existe un registro de estos laboratorios en el país.

En el año 2003, el Ministerio de Salud 
Pública (MSP) expidió el Reglamento General del Sistema Nacional de Salud, que en su artículo 21 menciona los requisitos de licenciamiento de los prestadores: "para la actividad de proveedores públicos y privados del sistema, los servicios deberán cumplir las normas de licenciamiento y los estándares mínimos definidos por el Ministerio de Salud Pública". ${ }^{7}$ En el 2005 se inició el licenciamiento de los servicios de salud en varias unidades operativas del MSP, hecho que ha permitido analizar y discutir la aplicabilidad de los mínimos estándares de calidad propuestos en el proceso de licenciamientos, que aseguran la protección de la salud y la seguridad pública.

Tomando en cuenta estos alcances, consideramos importante llevar a cabo un diagnóstico situacional de los laboratorios clínicos para evaluar el sistema de registro de los mismos, el recurso humano que allí labora y su idoneidad. Este diagnóstico permitirá a un futuro tomar acciones y estrategias que mejoren el sistema de vigilancia de los laboratorios clínicos del Ecuador, hecho que permitirá optimizar la aplicabilidad de los reglamentos expedidos en la Ley del Sistema Nacional de Salud y el Código de la Salud del Ecuador.

El presente estudio tuvo la colaboración del Proceso de Control y Mejoramiento en Gestión de Servicios de Salud del Ministerio de Salud Pública del Ecuador, la Maestría en Salud Pública de la Universidad San Francisco de Quito y la Confederación Latinoamericana de Bioquímica Clínica (COLABIOCLI).

\section{OBJETIVOS}

\subsection{General}

Diagnóstico situacional de los laboratorios de diagnóstico clínico en el país.

\subsection{Objetivos Específicos}

- Determinar el número de laboratorios de diagnóstico clínico en el país.

- Determinar el número y tipo de profesionales responsables que laboran en dichos laboratorios.

- Comprobar la existencia de información adecuada a nivel catastral con relación a los laboratorios de diagnóstico clínico en el país.

\section{MÉTODOS E INSTRUMENTOS}

El presente diagnóstico situacional consideró una serie de variables basados en experiencias de otros países y que se destacan a continuación ${ }^{8}$ :

- Sistema de registros de los laboratorios clínicos.

- Compromiso de las autoridades de salud en el cumplimiento de las políticas de salud relevantes al tema.

- Formación científica del personal de laboratorio.

- Coordinación entre las Direcciones Provinciales de Salud y el Proceso de Control y Mejoramiento en Gestión de Servicios de Salud del Ministerio de Salud Pública.

\subsection{Selección de la muestra}

Se consideró a todas las 21 provincias del país, de las cuales se solicitó la información que se describe posteriormente.

Para alcanzar los objetivos señalados se 
plantearon 4 etapas:

\section{Etapa de Información}

Se envió una circular donde se informó a las 21 Direcciones Provinciales de Salud del país acerca de los objetivos planteados en el proyecto, acompañada de un Formulario de Recolección de Datos de Laboratorio que sirvió para que las Direcciones recopilen la información necesaria para efectuar el diagnóstico situacional. Posteriormente se realizó un seguimiento a través de llamadas telefónicas a las direcciones. En el Formulario de Recolección de Datos de Laboratorio se solicitó el envío de la siguiente información:

1. Número de laboratorios de diagnóstico clínico existentes en la provincia.

2. Número de laboratorios de diagnóstico clínico con permiso de funcionamiento/2004.

3. Número de laboratorios de diagnóstico clínico que funcionan en servicios de salud privados: hospitales, clínicas, centros médicos.

4. Número de laboratorios de diagnóstico clínico que cuentan con profesionales responsables, como médicos especialistas en Patología Clínica/Laboratorio Clínico.

5. Número de laboratorios de diagnóstico clínico que cuentan con profesionales responsables, como médicos especialistas en Anatomía Patológica.

6. Número de laboratorios de diagnóstico clínico que cuentan con profesionales responsables, como Bioquímicos Farmacéuticos o Químicos Farmacéuticos.

7. Número de laboratorios de diagnós- tico clínico que cuentan con profesionales de apoyo, como Lcdos. en Laboratorio Clínico.

8. Número de laboratorios de diagnóstico clínico que cuentan con profesionales de apoyo, como Tecnólogos Médicos.

Además se solicitó el envío de la información de Catastro que incluye lo siguiente:

1. Código de inscripción del Laboratorio Clínico

2. Razón Social

3. Nombre del Representante Profesional

4. Tipo de profesional responsable

5. Número de RUC

6. Fecha de inscripción

7. Dirección/ teléfono

\section{Etapa de recolección}

Se adjuntaron todos los catastros y Formularios de las diferentes Direcciones Provinciales de Salud, desde enero de 2005 hasta diciembre de 2005 y se los envió al Proceso de Control y Mejoramiento en Gestión de Servicios de Salud del Ministerio de Salud Pública del Ecuador.

\section{Etapa de procesamiento}

Los datos obtenidos fueron introducidos en una base de datos creada para el efecto en el paquete informático SPSS, para luego ser analizados en el mismo programa en base a un plan de análisis de tipo descriptivo en el que se utilizaron frecuencias y porcentajes.

Informe final 
Se redactó un informe en el que se incluyeron los principales hallazgos.

\section{RESULTADOS}

La Tabla 1 muestra las Direcciones Provinciales de Salud que enviaron sus catastros y Formularios hasta el 15 de diciembre de 2005. Apenas 9 de las 21 provincias entregaron la información catastral oportunamente. Con relación al Formulario de Recolección de Datos, 14 de las 21 provincias los completaron y enviaron de vuelta. Solo cinco provincias enviaron la infor- mación tanto de catastro como del Formulario.

Las provincias de Esmeraldas, Los Ríos, Pichincha, Cañar y Orellana enviaron toda la información requerida, mientras que Carchi, Napo y Morona Santiago no enviaron ningún tipo de información (catastro ni Formulario) hasta diciembre de 2005.

En la Tabla 2 se muestra la información que registraron las Direcciones Provinciales de Salud en los catastros del 2004 y que fue enviada al Proceso de Control y Mejoramiento en Gestión de

Tabla 1. Provincias que enviaron información de catastros y Formularios de Recolección de Datos de Laboratorios Clínicos del Ecuador. 2005

\begin{tabular}{|c|c|c|}
\hline PROVINCIAS & $\begin{array}{c}\text { FORMULARIOS } \\
\text { ENVIADOS }\end{array}$ & $\begin{array}{l}\text { CATASTROS } \\
\text { ENVIADOS }\end{array}$ \\
\hline Esmeraldas & sí & sí \\
\hline Manabí & no & sí \\
\hline Guayas & Sí & no \\
\hline El Oro & sí & no \\
\hline Los Ríos & sí & si \\
\hline Carchi & no & no \\
\hline Imbabura & sí & no \\
\hline Pichincha & sí & sí \\
\hline Cotopaxi & sí & no \\
\hline Tungurahua & no & sí \\
\hline Bolívar & no & no \\
\hline Cañar & $\overline{\text { sí }}$ & sí \\
\hline Azuay & Sí & no \\
\hline Loja & Sí & no \\
\hline Chimborazo & no & Sí \\
\hline Sucumbíos & no & sí \\
\hline Zamora Chinchipe & sí & no \\
\hline Morona Santiago & sí & no \\
\hline Napo & no & no \\
\hline Orellana & sí & sí \\
\hline Galápagos & Sí & no \\
\hline TOTAL & 14 & 9 \\
\hline
\end{tabular}

Fuente: Catastros de DPS, M.S.P, 2004

Elaboración: Autores 
Servicios de Salud del Ministerio de Salud Pública.

Cabe destacar que la provincia de Los Ríos no registró ninguna de la información solicitada en los Catastros, aunque en general ninguna provincia proporcionó información catastral completa.

Las provincias de Manabí, Los Ríos, reportó el mayor número y porcentaje global de laboratorios de toda la muestra, (299 laboratorios, $78 \%$ del total de la muestra), seguido de lejos por Tungurahua con 28 laboratorios registrados en Catastro. Por otro lado, Los Ríos no registra laboratorios clínicos en el Catastro del 2004. (Tabla 3).

De un total de 1001 laboratorios regis-

Tabla 2. Información sobre los Laboratorios Clínicos del Ecuador que registraron las Direcciones Provinciales de Salud en los Catastros del 2004.

\begin{tabular}{|l|l|c|c|l|l|c|c|c|c|c|}
\hline $\mathbf{N}^{0}$. & Provincias & Código & $\begin{array}{l}\text { Razón } \\
\text { Social }\end{array}$ & $\begin{array}{l}\mathbf{N}^{0} . \\
\text { RUC }\end{array}$ & $\begin{array}{l}\text { Representante } \\
\text { Profesional }\end{array}$ & $\begin{array}{l}\text { Tipo de } \\
\text { Profesional }\end{array}$ & Dirección & Teléfono & $\begin{array}{l}\mathbf{N}^{0} \text {. Permiso } \\
\text { Anterior }\end{array}$ & $\begin{array}{l}\mathbf{N}^{0} \text { Permiso } \\
\text { Actual }\end{array}$ \\
\hline 1 & Esmeraldas & - & + & - & - & - & + & - & - & - \\
\hline 2 & Manabí & - & + & - & - & - & - & - & - & - \\
\hline 3 & Los Ríos & - & - & - & - & - & - & - & - & - \\
\hline 4 & Pichincha & - & + & - & + & + & + & - & - & - \\
\hline 5 & Tungurahua & - & + & - & + & + & + & - & - & - \\
\hline 6 & Chimborazo & + & + & + & + & - & + & - & - & + \\
\hline 7 & Cañar & + & + & + & + & - & + & + & + & + \\
\hline 8 & Sucumbíos & - & + & + & + & - & + & - & - & - \\
\hline 9 & Orellana & - & + & + & - & + & + & - & - & + \\
\hline
\end{tabular}

Fuente: Catastros de DPS, M.S.P, 2004

Elaboración: Autores

Esmeraldas, Orellana, no registraron el nombre del profesional responsable de los laboratorios clínicos. Ninguna provincia, excepto Cañar, registró el número de teléfono de los laboratorios clínicos en los Catastros. La mayoría de provincias no registraron el número anterior y el número actual de permiso de funcionamiento. Es importante recalcar que varias provincias como Guayas, El Oro, Loja, Zamora Chinchipe, Morona Santiago y Galápagos no enviaron el Catastro, pero sí enviaron el Formulario.

La Provincia de Pichincha fue la que trados en los Formularios de Recolección de Datos, 452 (46\%) no contaban con permiso de funcionamiento. En la Tabla 4 se observa que en cuatro de 14 provincias $(28 \%)$, el porcentaje de laboratorios sin permiso de funcionamiento fue igual o mayor a 50\% (Los Ríos, Pastaza, Esmeraldas, y Guayas). Las provincias que más cumplían con este requisito fueron Galápagos (100\%), Azuay $(95 \%)$ e Imbabura (85\%).

Al comparar la información proveniente del catastro y del Formulario de Recolección de Datos en las cinco provincias que entregaron los dos instrumentos, 
Tabla 3. Número de Laboratorios Clínicos registrados en los Catastros del 2004, en 9 provincias del Ecuador.

\begin{tabular}{|cl|c|c|}
\hline $\mathbf{N}^{\mathbf{o}}$. & PROVINCIAS & $\begin{array}{l}\mathbf{N}^{\mathbf{o}} \text { Laboratorios } \\
\text { Clínicos }\end{array}$ & Porcentaje \\
\hline 1 & Esmeraldas & 5 & 1,31 \\
\hline 2 & Manabí & 17 & 4,45 \\
\hline 3 & Los Ríos & 0 & 0,00 \\
\hline 4 & Pichincha & 299 & 78,27 \\
\hline 5 & Tungurahua & 28 & 7,33 \\
\hline 6 & Chimborazo & 7 & 1,83 \\
\hline 7 & Cañar & 20 & 5,24 \\
\hline 8 & Sucumbíos & 1 & 0,26 \\
\hline 9 & Orellana & 5 & 1,31 \\
\hline \multicolumn{2}{|c|}{ TOTAL } & $\mathbf{3 8 2}$ & $\mathbf{1 0 0}$ \\
\hline
\end{tabular}

Fuente: Catastros de DPS, MSP, 2004

Elaboración: Autores

se puede observar que existió una diferencia de 110 laboratorios (33\%), entre los registrados en Catastro (329) y los laboratorios con permiso de funcionamiento registrados en los Formularios (219). En algunas provincias existen grandes diferencias entre el número de laboratorios registrados en el catastro y los que se encuentran en el Formulario. Los Ríos no registró ningún laboratorio en el catastro, aunque en el Formulario se registraron 16 (ver Tabla 5). El número de laboratorios clínicos registrados en los Formularios tiende a ser mayor que los reportados en los catastros, con la excepción de Pichincha.

La Tabla 6 muestra las provincias que enviaron información del número de laboratorios clínicos ubicados dentro de clínicas, hospitales y centros médicos privados, datos que se registraron en el Formulario de Recolección de Datos. Asumiendo que los laboratorios clínicos ubicados en los servicios de salud privados cuentan con permiso de funcionamiento, su número (93) representó el 76,2\% del total de los laboratorios con permiso de funcionamiento en las provincias que respondieron a esta pregunta. En Los Ríos y Esmeraldas el número reportado de laboratorios dentro de servicios de salud privados fue mayor que el total de laboratorios con permiso de funcionamiento reportado en los Formularios.

El Formulario de Recolección de Datos nos permitió recabar información referente al número de profesionales responsables de los laboratorios clínicos, es decir aquellos que tienen el perfil adecuado (médicos patólogos, anátomo-patólogos, bioquímicos farmacéuticos y químicos farmacéuticos). Guayas tuvo el mayor porcentaje de profesionales con el perfil adecuado para manejar laboratorios clínicos $(24,6 \%)$, seguido de cerca por Pichincha $(24,3 \%)$. El Oro sumó $14 \%$ de profesionales bioquímicos, exclusivamente. Orellana no cuenta 
Tabla 4. Laboratorios Clínicos existentes en las provincias con o sin permiso de funcionamiento, de acuerdo al Formulario de Recolección de Datos.

\begin{tabular}{|l|c|c|c|}
\hline Provincias & $\begin{array}{c}\text { Laboratorios clínicos } \\
\text { existentes en la } \\
\text { provincia según los } \\
\text { Formularios }\end{array}$ & $\begin{array}{c}\text { Laboratorios clínicos } \\
\text { CON permiso de } \\
\text { funcionamiento según } \\
\text { los Formularios }\end{array}$ & $\begin{array}{c}\text { Laboratorios Clínicos } \\
\text { SIN permiso de } \\
\text { funcionamiento según } \\
\text { los Formularios }\end{array}$ \\
\hline Los Ríos & 54 & $16(30 \%)$ & $38(70 \%)$ \\
\hline Pastaza & 12 & $6(50 \%)$ & $6(50 \%)$ \\
\hline Cañar & 28 & $22(78 \%)$ & $6(22 \%)$ \\
\hline Esmeraldas & 59 & $10(17 \%)$ & $49(83 \%)$ \\
\hline Orellana & 7 & $5(71 \%)$ & $2(29 \%)$ \\
\hline El Oro & 98 & $60(61 \%)$ & $38(39 \%)$ \\
\hline Imbabura & 30 & $25(83 \%)$ & $5(17 \%)$ \\
\hline Cotopaxi & $83(43 \%)$ \\
\hline Galápagos & 74 & $19(57 \%)$ & $0(0 \%)$ \\
\hline Loja & 110 & $8(100 \%)$ & $21(29 \%)$ \\
\hline Azuay & 262 & $53(71 \%)$ & $5(5 \%)$ \\
\hline Pichincha & 9 & $105(95 \%)$ & $96(37 \%)$ \\
\hline Zamora Chinchipe & 217 & $166(63 \%)$ & $4(45 \%)$ \\
\hline Guayas & $\mathbf{1 0 0 1}$ & $49(22 \%)$ & $168(78 \%)$ \\
\hline TOTAL & $\mathbf{5 4 9 ( 5 4 \% )}$ & $\mathbf{4 5 2 ( 4 6 \% )}$ \\
\hline
\end{tabular}

Fuente: Formularios de DPS, MSP, 2004

Elaboración: Autores

con ninguno. Los Bioquímicos fueron los profesionales que se reportaron en mayor número (79\% del total de 14 provincias) (ver Gráfico 1).

Cuando se analizó el porcentaje de laboratorios que contaban únicamente con personal de apoyo como responsables de su manejo (Licenciados en Laboratorio Clínico y Tecnólogos Médicos), la provincia del Azuay fue la que reportó el mayor número de laboratorios con este tipo de profesionales $(30 \%)$, seguido de Guayas $(17,8 \%)$, Los Ríos (10\%) y Esmeraldas $(9,3 \%)$.

La Tabla 9 muestra el número total de laboratorios clínicos en las 14 provincias del Ecuador, con los diferentes tipos de profesionales a cargo de estos laboratorios. Los bioquímicos representaron los profesionales más reportados a cargo de laboratorios clínicos, seguidos de los licenciados en laboratorio clínico (26.4\%).

\section{DISCUSIÓN}

Apenas 9 Direcciones Provinciales de Salud acataron la solicitud de información de catastros y 14 llenaron el Formulario de Recolección de Datos. Las provincias de Imbabura, Cotopaxi y Bolívar enviaron sus catastros, sin embargo no se accedió a esta 
Tabla 5. Laboratorios Clínicos registrados en los catastros del 2004 y Laboratorios Clínicos que constan en los Formularios de Recolección de Datos en cinco Direcciones Provinciales de Salud.

\begin{tabular}{|c|l|c|c|}
\hline $\mathbf{N}^{\mathbf{o}}$ & Provincias & $\begin{array}{c}\mathbf{N}^{\mathbf{o}} \text { Laboratorios Clínicos } \\
\text { registrados en los catastros }\end{array}$ & $\begin{array}{c}\mathbf{N}^{\mathbf{0}} \text { De Laboratorios Clínicos con permiso de } \\
\text { funcionamiento registrados en los Formularios }\end{array}$ \\
\hline 1 & Esmeraldas & $5(1,5 \%)$ & $10(4,6 \%)$ \\
\hline 2 & Los Ríos & 0 & $16(7,3 \%)$ \\
\hline 3 & Pichincha & $299(90,9 \%)$ & $166(75,8 \%)$ \\
\hline 4 & Cañar & $20(6 \%)$ & $22(10 \%)$ \\
\hline 5 & Orellana & $5(1,5 \%)$ & $5(2,3 \%)$ \\
\hline & TOTAL & $\mathbf{3 2 9}(\mathbf{1 0 0 \%})$ & $\mathbf{2 1 9}(\mathbf{1 0 0 \%})$ \\
\hline
\end{tabular}

Fuente: Formularios de DPS, MSP, 2004

Elaboración: Catastros de DPS, MSP, 2004

información por problemas de virus informático en la base de datos. Apenas cinco provincias enviaron la información completa tanto de Formularios como catastros, como se menciona en la Tabla 2 (Los Ríos no registró ninguna información en los catastros del 2004), lo que muestra ya sea la inexistencia de dicha información, una falta de recursos para obtenerla, o una falta de compromiso por parte de los responsables provinciales para recabarla. Adicionalmente, de entre aquellas provincias que sí enviaron la información catastral, se comprobó que ésta no está siendo registrada de manera óptima en la mayoría de los casos.

Llama la atención que muchos de los laboratorios clínicos en las diferentes provincias no contaban con permiso de funcionamiento: el $46 \%$ de los laboratorios clínicos de las 14 provincias que enviaron información, no tenían permiso de funcionamiento. La provincia con el mayor número de laboratorios clínicos sin permiso de funcionamientos fue Guayas (78\%), mientras que Galápagos registró el mayor porcentaje de laboratorios clínicos con dicho permiso. Este requisito debe ser considerado como indispensable para garantizar un mínimo nivel de control de calidad.

Se observó una tendencia a que el número de laboratorios clínicos con permiso de funcionamiento registrados en el Formulario de Recolección de Datos sea mayor al registrado en el catastro, con la excepción de la provincia de Pichincha. Se esperaría a que estos datos fueran similares, ya que los laboratorios que constan en el catastro son aquellos que cuentan con permiso de funcionamiento. El número total de laboratorios clínicos en las 5 provincias que enviaron tanto el catastro como el Formulario fue de 329, lo que difiere con el Formulario donde se reportaron 219 laboratorios clínicos con permiso de funcionamiento. En el caso de la provincia de Los Ríos, el Formulario registró 16 laboratorios clínicos con permiso de funcionamiento, sin embargo en los catastros no se evidenció la existencia de ningún laboratorio. Posiblemente existen deficiencias en la recolección de información catastral o la información para el Formulario no fue recopilada de una manera técnica.

En el caso de la provincia de Pastaza, 
Tabla 6. Laboratorios Clínicos que se ubican dentro de servicios de salud registrados en los Formularios de Recolección de Datos.

\begin{tabular}{|c|c|}
\hline PROVINCIAS & $\begin{array}{c}\mathbf{N}^{\mathbf{o}} \text { de Laboratorios Clínicos } \\
\text { registrados en los Formularios }\end{array}$ \\
\hline Los Ríos & 36 \\
\hline Pastaza & 6 \\
\hline Cañar & 7 \\
\hline Esmeraldas & 21 \\
\hline Orellana & 3 \\
\hline El Oro & 1 \\
\hline Imbabura & NO CONTESTA \\
\hline Cotopaxi & NO CONTESTA \\
\hline Galápagos & NO CONTESTA \\
\hline Loja & NO CONTESTA \\
\hline Azuay & NO CONTESTA \\
\hline Pichincha & 4 \\
\hline Zamora Chinchipe & 10 \\
\hline Guayas & $\mathbf{9 3}$ \\
\hline TOTAL & \\
\hline
\end{tabular}

Fuente: Formularios de DPS, MSP, 2004

Elaboración: Autores

los únicos laboratorios registrados fueron los ubicados en servicios de salud privados. En Esmeraldas y Los Ríos llamó la atención que existían más laboratorios ubicados en servicios de salud (21) que todos los reportados en el Formulario y que contaban con permiso de funcionamiento. Esto puede significar que quizás existirían laboratorios en servicios de salud privados que no cuentan con el respectivo permiso o que la información en el Formulario es errada. El Oro registró apenas 1 laboratorio dentro de un servicio de salud privado. Llama la atención que Los Ríos tenga el mayor número de estos laboratorios institucionales (36), a pesar de no registrar ningún laboratorio con permiso de funcionamiento.

Con relación a los profesionales con el perfil adecuado responsables de los laboratorios clínicos, como era de esperarse, el mayor porcentaje (49\%) se encontró en las provincias de Guayas y Pichincha, regiones de mayor concentración de personal calificado. En general, los bioquímicos fueron los profesionales con perfil adecuado a cargo de la mayoría de laboratorios clínicos de las provincias del estudio, aunque preocupa el hecho de que muchos laboratorios eran manejados por personal de apoyo.

En la mayoría de provincias que enviaron información existió un mayor porcentaje de personal de apoyo a cargo de los laboratorios clínicos, comparado con los profesionales con el perfil adecuado. Azuay $(30 \%)$ tuvo el mayor porcentaje de personal de apoyo a cargo de laboratorios clínicos, mientras que sólo el $15 \%$ de los labo- 
Tabla 7. Laboratorios Clínicos que cuentan con profesionales responsables de laboratorio, en 14 provincias del Ecuador.

\begin{tabular}{|l|c|c|c|c|c|}
\hline PROVINCIAS & $\begin{array}{c}\text { Número de } \\
\text { laboratorios con } \\
\text { PATÓLOGOS } \\
\text { CLÍNICOS }\end{array}$ & $\begin{array}{c}\text { Número de } \\
\text { laboratorios con } \\
\text { ANATOMÍA } \\
\text { PATOLÓGICA }\end{array}$ & $\begin{array}{c}\text { Número de } \\
\text { laboratorios con } \\
\text { BIOQUÍMICOS- } \\
\text { FARMACÉUTICOS } \\
\text { /QUÍMICOS } \\
\text { FARMACÉUTICOS }\end{array}$ & TOTAL & $\begin{array}{c}\text { PORCENTAJE } \\
\text { TOTAL }\end{array}$ \\
\hline Los Ríos & $5(28 \%)$ & $0(0 \%)$ & $13(72 \%)$ & 18 & 2,63 \\
\hline Pastaza & $0(0 \%)$ & $0(0 \%)$ & $4(100 \%)$ & 4 & 0,58 \\
\hline Cañar & $1(4 \%)$ & $0(0 \%)$ & $26(96 \%)$ & 27 & 3,95 \\
\hline Esmeraldas & $10(40 \%)$ & $5(20 \%)$ & $10(40 \%)$ & 25 & 3,65 \\
\hline Orellana & $0(0 \%)$ & $0(0 \%)$ & $0(0 \%)$ & 0 & 0,00 \\
\hline El Oro & $0(0 \%)$ & $0(0 \%)$ & $97(100 \%)$ & 97 & 14,18 \\
\hline Imbabura & $3(25 \%)$ & $0(0 \%)$ & $9(75 \%)$ & 12 & 1,75 \\
\hline Cotopaxi & $1(13 \%)$ & $0(0 \%)$ & $7(88 \%)$ & 8 & 1,17 \\
\hline Galápagos & $0(0 \%)$ & $5(100 \%)$ & $0(0 \%)$ & 5 & 0,73 \\
\hline Loja & $20(42 \%)$ & $13(27 \%)$ & $15(31 \%)$ & 48 & 7,02 \\
\hline Azuay & $9(9 \%)$ & $8(8 \%)$ & $87(84 \%)$ & 104 & 15,20 \\
\hline Pichincha & $39(23 \%)$ & $2(1 \%)$ & $125(75 \%)$ & 166 & 24,27 \\
\hline Zamora Chinchipe & $1(50 \%)$ & $0(0 \%)$ & $1(50 \%)$ & 2 & 0,29 \\
\hline Guayas & $24(14 \%)$ & $0(0 \%)$ & $144(86 \%)$ & 168 & 24,56 \\
\hline TOTAL & $\mathbf{1 1 3 ( 1 6 , 5 \% )}$ & $\mathbf{3 3 ( 5 \% )}$ & $\mathbf{5 3 8 ( 7 9 \% )}$ & $\mathbf{6 8 4}$ & $\mathbf{1 0 0}$ \\
\hline
\end{tabular}

Fuente: Formularios de DPS, MSP, 2004

Elaboración: Autores

ratorios estaban a cargo de un profesional con el perfil adecuado.

\section{CONCLUSIONES}

1. No existe una información adecuada en varias de las Direcciones Provinciales de Salud que participaron en el estudio, en relación a los laboratorios clínicos de dichas provincias.

2. La falta de información podría deberse a deficiencias en el reporte por parte de los laboratorios, deficiencias en el registro por parte de la DPS o carencia de recursos para recopilar dicha información.

3. Existe una falta de recursos y de compromiso por parte de ciertas autoridades sanitarias con las políticas nacionales de salud, encaminadas a garantizar la calidad de los servicios de salud incluyendo los laboratorios clínicos.

4. El mecanismo actual del sistema de información de las Direcciones Provinciales de Salud (envío de catastros a través de un diskette al Proceso de Control y Mejoramiento en Gestión de Servicios de Salud del MSP), dificulta desarrollar y optimizar la infor- 
Gráfico 1. Porcentaje de profesionales, en relación a los tipos de profesionales responsables de los Laboratorios Clínicos en 16 provincias del Ecuador.

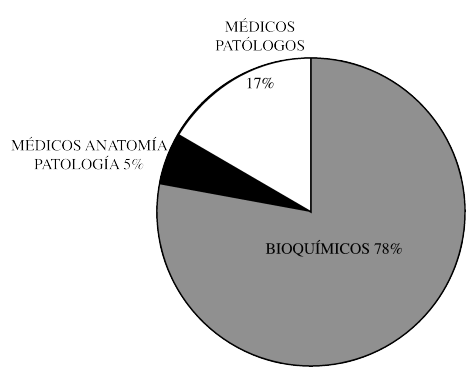

mación en relación al registro de datos, el adecuado procesamiento en el análisis y la transmisión de los resultados obtenidos de los laboratorios clínicos, hecho que impide mejorar la gestión a ese nivel.

5. El Ministerio de Salud Pública no cuenta con una red de información intrainstitucional eficiente en el caso de los laboratorios clínicos. El Proceso de Control y Mejoramiento en Gestión de Servicios de Salud, no registra el número de laboratorios clínicos públicos del país. Esta información está disponible en formato duro en el Departamento de Estadística, lo que dificulta un acceso oportuno y eficaz.

6. No existe un adecuado seguimiento de los laboratorios clínicos ubicados dentro de Clínicas, Hospitales, Centros Médicos privados de las provincias que reportaron información y se desconoce el número de laboratorios clínicos dentro de estos servicios de salud.

7. Se evidencia un incumplimiento del marco legal en relación al buen funcionamiento de los laboratorios clíni- cos del país, ya que un importante porcentaje no tiene permiso de funcionamiento.

8. Existe una mayor concentración de profesionales responsables de los laboratorios clínicos (médicos patólogos y bioquímicos) en las provincias más grandes y pobladas del país, (Guayas, Pichincha y Azuay), mientras que en el resto de provincias su presencia es baja.

9. Existen profesionales de apoyo dirigiendo laboratorios clínicos en varias provincias del país, aunque por su competencia no deberían hacerlo.

10. El mayor número de laboratorios clínicos habilitados está dirigido por profesionales Bioquímicos-Farmacéuticos y Químicos Farmacéuticos.

11. Los laboratorios clínicos sin permiso de funcionamiento son laboratorios dirigidos posiblemente por profesionales de apoyo (Licenciados en laboratorio clínico y Tecnólogos Médicos).

\section{RECOMENDACIONES}


Tabla 8. Laboratorios Clínicos que cuentan sólo con profesionales de apoyo, (Licenciados en Laboratorio Clínico y Tecnólogos Médicos) en 14 provincias del Ecuador.

\begin{tabular}{|l|c|c|c|c|}
\hline PROVINCIAS & $\begin{array}{c}\mathbf{N}^{\mathbf{0}} \text { Lab. con } \\
\text { Lcdos. en } \\
\text { Laboratorio } \\
\text { Clínico }\end{array}$ & $\begin{array}{c}\mathbf{N}^{\mathbf{0}} \text { Laboratorio } \\
\text { con } \\
\text { Tecnólogos } \\
\text { Médicos }\end{array}$ & TOTAL & PORCENTAJE \\
\hline Los Ríos & 16 & 21 & 37 & 10,11 \\
\hline Pastaza & 12 & 0 & 12 & 3,28 \\
\hline Cañar & 1 & 7 & 8 & 2,19 \\
\hline Esmeraldas & 4 & 30 & 34 & 9,29 \\
\hline Orellana & 5 & 2 & 7 & 1,91 \\
\hline El Oro & 4 & 17 & 21 & 5,74 \\
\hline Imbabura & 9 & 9 & 18 & 4,92 \\
\hline Cotopaxi & 11 & 11 & 22 & 6,01 \\
\hline Galápagos & 1 & 1 & 2 & 0,55 \\
\hline Loja & 13 & 13 & 26 & 7,10 \\
\hline Azuay & 10 & 100 & 110 & 30,05 \\
\hline Pichincha & 0 & 0 & 0 & 0,00 \\
\hline Zamora Chinchipe & 2 & 2 & 4 & 1,09 \\
\hline Guayas & 0 & 65 & 65 & 17,76 \\
\hline TOTAL & $\mathbf{8 8}$ & $\mathbf{2 7 8}$ & $\mathbf{3 6 6}$ & $\mathbf{1 0 0}$ \\
\hline
\end{tabular}

Fuente: Formularios de DPS, MSP, 2004

Elaboración: Autores

1. El marco legal existente referente al control y vigilancia de los laboratorios clínicos del país no se lo cumple a cabalidad. Se debe analizar, evaluar y mejorar el actual reglamento de funcionamiento de los laboratorios clínicos, considerando los datos proporcionados en la presente investigación, con la participación de un grupo interdisciplinario integrado por Asociaciones Gremiales, Ministerio de Salud Pública, Universidades y organismos interesados en mejorar la calidad de los laboratorios clínicos del país.

2.- La información aquí proporcionada deberá entrar en un proceso de revisión a nivel de la Dirección General de Salud, Direcciones Provinciales de Salud y Proceso de Control y Mejoramiento en Gestión de Servicios de Salud. Esto permitirá analizar el impacto que ha tenido el actual sistema de información en la calidad de los laboratorios clínicos del país, el desarrollo de los procedimientos dentro del Proceso de Control y Mejoramiento en Gestión de Servicios de Salud para laboratorios clínicos, y la utilidad del actual sistema de información para la toma de decisiones.

3.- El nivel central del MSP debe contactar a las Direcciones Provinciales de Salud 
Tabla 9. Profesionales en el área de Laboratorio Clínico registrados en el Formulario deRecolección de Datos.

\begin{tabular}{|l|r|r|}
\hline $\begin{array}{c}\text { Tipo de profesionales a cargo de } \\
\text { laboratorio }\end{array}$ & $\mathbf{N}^{\mathbf{o}}$ & Porcentaje \\
\hline Patólogos & 113 & 10,7 \\
\hline Anátomo-Patólogos & 33 & 3,1 \\
\hline Bioquímicos-Farmacéuticos & 538 & 51,0 \\
\hline Tecnólogos Médicos & 88 & 8,3 \\
\hline Lcds. Laboratorio Clínico & 278 & 26,4 \\
\hline TOTAL & $\mathbf{1 0 5 0}$ & $\mathbf{1 0 0 \%}$ \\
\hline
\end{tabular}

Fuente: Formularios de DPS, MSP, 2004

Elaboración: Autores

que no enviaron ninguna información tanto de catastros y Formularios, con el fin de evaluar el sistema de información que se viene utilizando en las mismas, evidenciar los registros de los laboratorios clínicos en situ y optimizar el sistema de información.

4. Implementar un sistema de información automatizado en las Direcciones Provinciales de Salud y una red de información intrainstitucional. Esto mejorará considerablemente la utilización de los recursos, agilitará la comunicación de las solicitudes y sus resultados, tanto de las Direcciones Provinciales de Salud y del Proceso de Control y Mejoramiento en Gestión de Servicios de Salud. De igual manera este sistema permitirá tener una información más completa, lo que representará una mayor cantidad de información disponible para la toma de las decisiones.

5. El Proceso de Control y Mejoramiento en Gestión de Servicios de Salud del Ministerio de Salud Pública del Ecuador debe buscar que el $100 \%$ de los laboratorios clínicos cuenten con el respectivo permiso de funcionamiento. Este requisito permitirá contar con una base de datos reales que permita un control de calidad en los laboratorios clínicos del país.

6. Debido a la carencia de personal adecuado a cargo de laboratorios clínicos en varias de las provincias del país, se debería estudiar la posibilidad de que los profesionales bioquímicos y químicos farmacéuticos realicen el año de trabajo rural, como lo hacen otras profesiones de salud en el país.

7. Se recomienda que el MSP realice un censo de laboratorios clínicos en todo el país, tanto de los independientes como de aquellos que funcionan dentro de un servicio de salud. 
Gráfico 2. Porcentaje de los diferentes tipos de profesionales en los Laboratorios Clínicos en las 14 provincias del Ecuador.

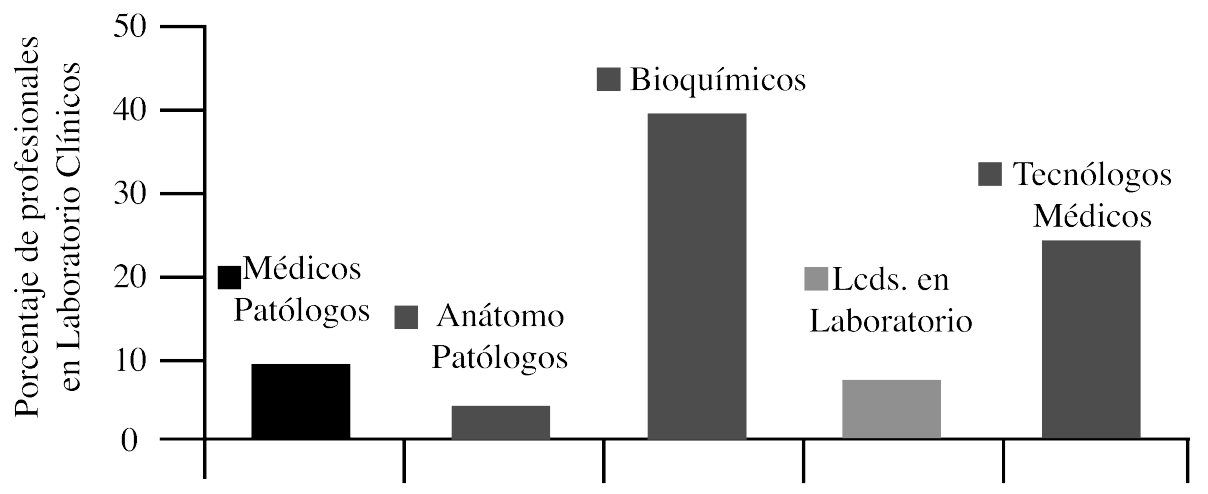

Tipo de Profesionales en Laboratorio Clínico

Fuente: Formularios de DPS, MSP, 2004

Elaboración: Autores 